\title{
Regulación por Privatización de Recursos Naturales de Uso Común
}

\author{
Regulación por Privatización de Recursos Naturales de Uso Común
}

\author{
Laura Milena Cárdenas ${ }^{1}$, Yony Fernando Ceballos ${ }^{2}$, Jorge Parra ${ }^{3}$, Luis Eduardo Muñoz ${ }^{4}$, \\ ${ }^{I}$ Departamento de ciencias de la computación y la decisión, Universidad Nacional de Colombia, Medellín, Colombia \\ lauracaregmail. com \\ ${ }^{2}$ Departamento de Ingeniería Industrial, Facultad de Ingeniería, Universidad de Antioquia UdeA, Medellín, Colombia \\ yony. ceballos@udea.edu.co \\ ${ }^{3}$ Programa de Ingeniería de Sistemas, Universidad Autónoma de Bucaramanga, Bucaramanga, Colombia \\ japarra@unab.edu. co \\ ${ }^{4}$ Faculta de ingenierías, Ingeniería de Sistemas y Computación, Universidad Tecnológica de Pereira, Pereira, Colombia \\ lemunozg@utp.edu.co
}

\begin{abstract}
Resumen- Este artículo aborda la problemática referente a la regulación de los recursos naturales de uso común, evaluando dinámico sistémicamente el mecanismo de la privatización, a partir de la formulación de hipótesis dinámicas. Se considera pertinente establecer una investigación, que permita iluminar la siguiente pregunta de: ¿Cómo es que un país tan rico puede depredar sus recursos comunes, y será posible explicar esta situación evaluando a través del modelamiento con Dinámica de Sistemas del mecanismo de regulación de la privatización nivel genérico y a nivel de un caso específico?
\end{abstract}

Un paso inicial que se dio para tratar de abordar la pregunta de investigación planteada en este artículo, es poder indagar las implicaciones dinámicas mediante el diseño de un modelo dinámico sistemico, para el mecanismo de la privatización, en dos niveles de complejidad conceptual: uno genérico y uno específico a un recurso natural común, realizando experimentos que permitan ver si las teoría de regulación explica o no explica la preocupante situación que abarca el recurso tratado en Colombia.

Índice de Términos- Depredación de recursos naturales de uso común, Mecanismo de privatización, Tragedia del terreno común.

Abstract - This article discusses the problems concerning the regulation of natural resources commonly used dynamic systemically evaluated the mechanism of privatization, from the formulation of dynamic hypotheses. It is considered appropriate to establish an investigation, in order to illuminate the following question: How is it that a country so rich can plunder their common resources, and be able to explain this situation evaluated through system dynamics modeling with the mechanism of regulation privatization generic level and at the level of a specific case?
An initial step was given to try to address the research question in this article is to explore the dynamic implications by designing systemic dynamic model for the mechanism of privatization, at two levels of conceptual complexity: a generic and one specific to a common natural resource, conducting experiments to see if the theory of regulation explains or does not explain the worrying situation treaty covering the use in Colombia.

KeyPress- Predation of natural resources commonly used mechanism privatization, Tragedy of the Commons..

\section{INTRODUCCIÓN}

En esta investigación se abordaron tres afirmaciones que construyen las fronteras de la pregunta principal de investigación, a saber: la primera, en Colombia son abundantes los recursos comunes, tales como: los recursos hídricos, la fauna, la flora y sus bosques; la segunda, Colombia está obligada a preservar y distribuir equitativamente dichos recursos; entonces se esperaría que la conclusión lógica fuera, que Colombia preservara y distribuyera equitativamente dichos recursos, pero paradójicamente dicha conclusión no se cumple en la práctica las estadísticas hacen pensar que las políticas que aplica el Estado no están produciendo los resultados esperados, por lo que surge la pregunta de: ¿Cómo es que Colombia siendo un país tan rico en recursos comunes y teniendo la obligación de preservarlos, los depreda? 


\section{OBJETIVOS}

\section{A. Objetivo general}

Proponer una primera aproximación desde la Dinámica de Sistemas que permita evaluar la efectividad de la regulación por privatización en la mitigación de la depredación.

\section{B. Objetivos Específicos}

Diseñar un modelo Dinámico Sistémico para el mecanismo de regulación de la privatización a un nivel de abstracción genérico como explicación de su depredación. Realizar un modelo Dinámico Sistémico para el mecanismo de regulación de la privatización a un nivel de un caso de aplicación específica, que permita ilustrar una problemática colombiana en dicha área como explicación de su depredación en el país.

\section{METODOLOGIA}

Esta investigación se llevó a cabo en dos niveles: uno genérico y otro de un caso especifico. En cada uno de estos niveles se aplicaron las cuatro etapas propuestas: primera etapa la conceptualización, segunda etapa la formulación del modelo, tercera etapa la simulación del modelo y cuarta etapa la evaluación del modelo.

En la primera etapa del nivel del genérico, se realizó un levantamiento del estado del arte referente a la Dinámica de Sistemas y la regulación del uso de Recursos comunes.

En la segunda etapa, se llevaron a cabo los siguientes pasos:

En el primer paso se hizo la distinción del problema.

En el segundo paso se identificaron los factores causantes de que ocurra la inadecuada regulación del uso de recursos comunes en la sociedad.

En el tercer paso teniendo en cuenta los dos pasos anteriores, se establecieron los ciclos de realimentación de información causa-efecto, en donde lo que se pretende es relacionar las decisiones tomadas, con la acción que resulte del mismo, con el cambio de la información después de pasar por dichos ciclos y con las nuevas decisiones que se vayan a tomar.

En el cuarto paso se dio comienzo con la elaboración de un modelo matemático de las políticas de decisión obtenidas en el paso anterior, de fuentes informativas y de la iteración de los diferentes componentes que hacen parte del sistema.

En la tercera etapa, se realizó la simulación de dicho modelo, teniendo en cuenta que primero se debe generar el comportamiento del sistema con respecto al tiempo utilizando la simulación digital en Powersim.

En la cuarta etapa, se realizó una evaluación orientada a medir que tanto el modelo de la privatización explica la paradoja encontrada en esta investigación, para realizar una proyección de las implicaciones dinámico-sistémicas y comparar los resultados obtenidos en él con los datos empíricos.

En el segundo nivel del artículo se realizaron cada una de las fases, aplicadas a un caso específico colombiano, establecido previamente, el cual permita conocer si las explicaciones que ofrecen las simulaciones del mecanismo de regulación es una apropiada explicación de la paradoja que se ha propuesto: Colombia, siendo un país tan rico en recursos naturales y estando obligado por ley a preservarlos y distribuirlos, los depreda y los distribuye inadecuadamente.

\section{CONTENIDO}

Algunos conceptos se hacen presentes en el desarrollo de este artículo, éstos son:

Recurso común: Son aquellos que cumplen con 2 características: la primera de ellas, habla del hecho que deben ser no excluibles, es decir, que cualquier persona puede hacer uso de ellos, nadie puede hacer a un lado al otro por utilizarlos y la segunda dice, que deben presentar rivalidad, lo que significa que solo una persona o grupos de personas de entre todas las que pueden hacer uso de él, pueden tomarlo efectivamente, llegando al caso en que varias personas se adueñen del mismo y en su constante utilización lo agoten poco a poco. Algunos ejemplos de recursos comunes son: aire, agua, flora y fauna..

Tragedia de los comunes: Esta situación fue inicialmente tratada por William Foster Lloyd en 1833, para luego ser retomada públicamente por Garrett Hardin, quien por medio de su ensayo titulado "The Tragedy of commons", plantea que las personas que utilizan los recursos comunes están en medio de un proceso que termina en la sobreexplotación del recurso [1].

Mecanismos de regulación de recursos comunes: Frente a la problemática de la administración de los recursos comunes se han establecido tres posibles formas de gestión para los mismos: la primera es la regulación central por parte del estado, la segunda, a través de la privatización y la tercera, la organización y administración de las propias comunidades involucradas en el recurso. 
Privatización. Este mecanismo considera la asignación de ciertos derechos de propiedad privada a los individuos que se encuentran bajo la utilización del recurso común, de manera que cada una de las personas involucradas puedan establecer negocios con otras personas y de esta lograr mutuo beneficio para los involucrados, sin que la mayoría de las veces sea necesaria la intervención de terceros para su regulación, a esta solución se le denomina "Solución de la mano invisible" [2].

\section{RESULTADOS}

\section{A. Modelos genéricos}

Arquetipo del Mecanismo de Regulación de Privatización Ver apéndice 1.

Este mecanismo se basa en la división del recurso. Cada división es entregada a entidades privadas, quienes de forma independiente deben controlar el recurso asignado, entre ésto se incluye un mantenimiento continuo del recurso, con el fin de que se mantenga la sostenibilidad del mismo. Además se debe establecer una constante vigilancia y seguridad para evitar que la otra entidad haga una explotación ilegal sobre el recurso que se tiene esta entidad privada, ya que se puede dar el caso en el que para evitar costos y aumentar las ganancias no respeten las divisiones correspondientes. Finalmente cada entidad establece un control o monitoreo privado con el fin de revisar los niveles de extracción y apropiación ${ }^{1}$ del recurso [3].

Todos estos factores llevan consigo una serie de costos, que disminuyen las ganancias que pueden obtener las entidades privadas. Pero dichas ganancias a su vez se ven afectadas con una tasa de descuento para la apropiación del negocio.

En este mecanismo es importante resaltar que a pesar de que se establece una división equitativa del recurso, condiciones externas varían el comportamiento del recurso y puedan dar mayor rentabilidad hacia una de las entidades privadas, generando desigualdad entre ellas [3].

\section{B. Modelos específicos}

Ver apéndice 2 .

De acuerdo a la teoría sobre este mecanismo, se hace una división del recurso, por esta razón es que se presentan dos sistemas del recurso, cada uno representa la regulación que

\footnotetext{
${ }^{1}$ Terminología de Ostrom para referirse al proceso de sustracción del recurso. (Ostrom, 1990).
}

hace cada propiedad (en este caso, dos propiedades) del recurso.

El recurso se articula por varios ciclos, el primero de ellos, es el ciclo pequeño de bagre-nacimientos-bagre. En este ciclo se presenta el proceso por el cual se reproduce el bagre.

Pero la dinámica de apropiación del bagre también se articula por otro ciclo, bagre-muertes-bagre, a través de él disminuye la población de bagre, estas muertes son naturales, es decir, son causadas por efectos del medio y por características propias del ciclo de vida del bagre.

El apéndice 2 también presenta ciclos relacionados entre ellos pero que intervienen directamente con la población de bagre; el primero de ellos es pesca-ingresos-ganancias-pesca, en él se establece que a partir de la actividad de la pesca que se realice se generan unos ingresos que determinan el valor de las ganancias que condicionan a que se continúe realizando la pesca y el segundo es pesca-costos-ganancias-pesca, en él se establece que a partir de la pesca se producen unos costos que disminuyen o compensan ese valor de las ganancias. Estos ciclos srmiten establecer el valor de las ganancias (ya sea aumentándolo o disminuyéndolo) que a su vez es un factor determinante para determinar el valor de la pesca.

Los ciclos de apropiación junto con el ciclo de muerte natural, la mayoría de las veces son mayores que el ciclo de reposición del bagre, ocasionando una disminución cada vez mayor de la población del bagre, es decir, su sobreexplotación.

El ciclo que se encuentran representados por el color marrón en cada una de las propiedades, pesca-control-pesca, establece el control que considere necesario para regular la actividad de la pesca en su propiedad [4].

En el apéndice 2 también aparecen unas relaciones entre las dos propiedades, estas relaciones representan la pesca que hace cada una de las propiedades del bagre que no corresponde a su propiedad.

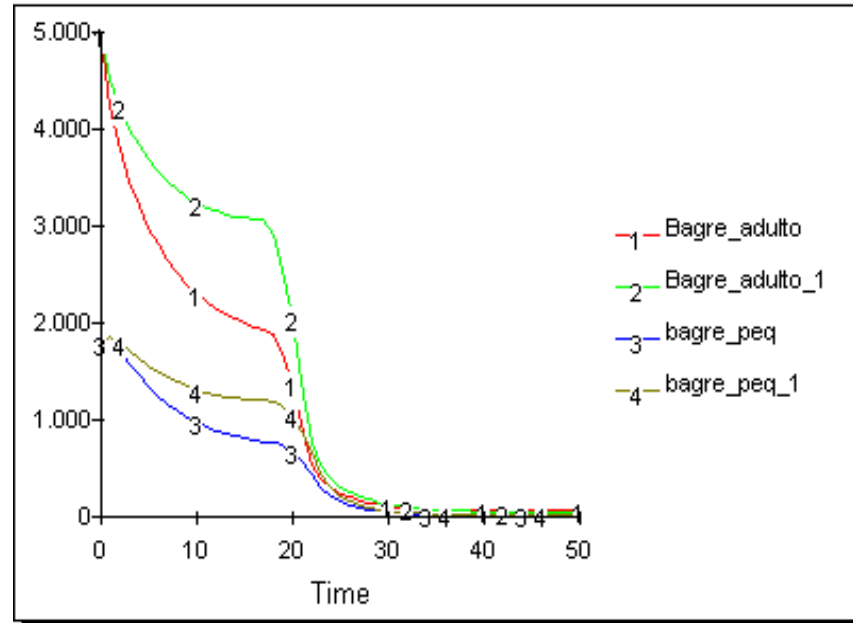


Figura 1. Simulación del bagre adulto y bagre pequeño de las propiedad 1 y 2 del modelo de regulación por privatización con superficies diferentes.

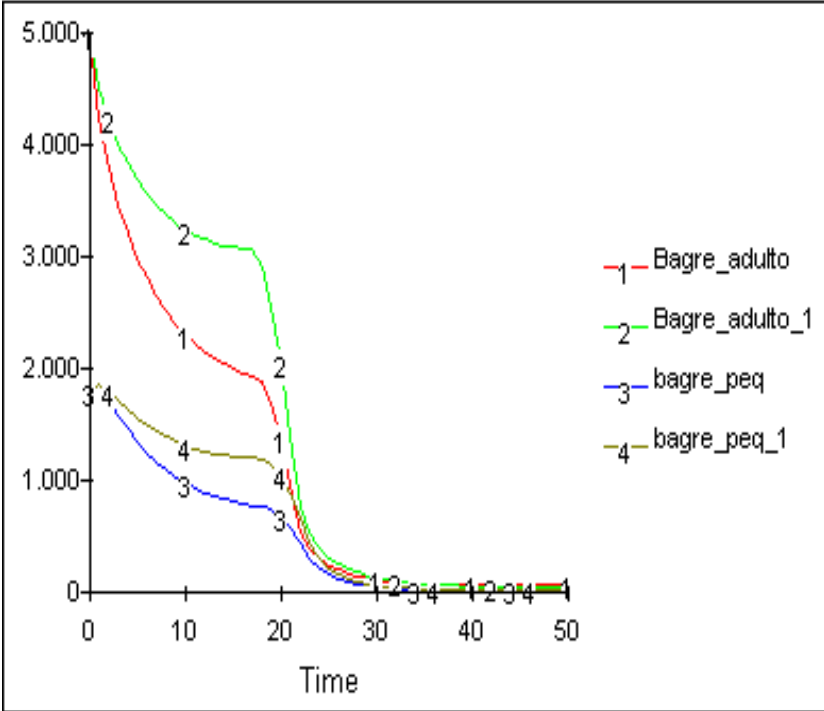

Figura 2. Simulación de las canoas de las propiedades 1 y 2 del modelo de regulación por privatización con superficies diferentes.

Para las simulaciones de este modelo se tuvo en cuenta que las superficies de las dos propiedades eran diferentes.

Cada una de estas propiedades se encarga de establecer el control para cada una de ellas, control que implica la vigilancia que cada una de éstas pueda hacer sobre la propiedad para que no se realicen dentro de ellas pescas ilegales (pescas de canoas de otras propiedades) y controlar las pescas de las canoas de la misma propiedad.

En la figura 1 se observa el comportamiento de las variables bagre adulto y pequeño de ambas propiedades, la propiedad 2 tiene una superficie más pequeña que la de la superficie 1 y los resultados de ella presentan valores más altos es decir en el apéndice 2 las líneas de color verde y marrón representan los niveles del bagre en la propiedad 2, aunque la propiedad 1 tiene una superficie mayor que la de la propiedad 1, ambas se ven envueltas en una dinámica de depredación debido a la influencia que ejercen las canoas (ver figura). Este número de canoas se reguló a través del control que cada una de las propiedades estableció, puesto que la propiedad 2 es mas pequeña se ejerció mayor control de la misma (el valor del control de la propiedad 2 es de 0.920 y el de la propiedad 1 es de 0.210 ), reflejado en las tasas de pesca ilegal tanto de bagre pequeño como bagre adulto.

Sin embargo es inevitable llevar a una sobreexplotación el bagre en ambas propiedades, ya que si bien cada una establece sus medidas de explotación del bagre, cada una quiere obtener el mayor número de ganancias y la manera de obtenerlo es a través de la pesca.

\section{CONCLUSIONES}

De acuerdo a la pregunta de investigación planteada en este proyecto de grado, ¿Cómo es que un país tan rico puede depredar sus recursos comunes? y ¿Será posible explicar esta paradójica situación evaluando a través del modelamiento con Dinámica de Sistemas del mecanismo de regulación del Estado a nivel genérico y a nivel de un caso específico? El estudio específico presentó más opciones para seguir trabajando en ella que el estudio genérico.

El mecanismo de la privatización, en las simulaciones realizadas no logró regular el recurso, se presentó una depredación del bagre puesto que al dividirse el recurso, los propietarios querrán sacar el mayor provecho de su propiedad debido a los costos ocasionados tanto por su mantenimiento como adquisición.

Debido a la complejidad que tiene llegar a estudiar sistemas sociales, en este caso conocer completamente la dinámica del bagre, se hace presente la duda durante todo el estudio de que tan precisos son los supuestos modelados y si logran reflejar completamente la situación del caso de estudio

La DS permitió llevar a cabo una explicación de cómo se entendía el fenómeno de la depredación en el recurso del bagre en el Banco del Magdalena a un nivel muy básico, ya que muchas expresiones de la teoría y conceptos son difíciles llegar a expresarlos en términos matemáticos que se escapan al nivel de abstracción que exige un modelo dinámico sistémico. Además el tiempo fue otro factor que no permitió extender la investigación, lo que llevó a limitar el alcance del mismo.

\section{REFERENCIAS}

[1] H. Garrett. "The Tragedy of the Commons". Science. Vol. 162. pp. 1245-1248. 1968.

[2] S, Adam. The Wealth of Nations. New York: Random House, 1937.

[3] E. Ostrom, "Governing the Commons: The evolution of institutions for collective action". Cambridge ; New York : Cambridge University Press, 1990. También publicado en español: Ostrom, Elinor (2000) "El Gobierno de los Bienes Comunes". Fondo de Cultura Económica y CRIM. Mexico, 2000 .

[4] L. Cárdenas y J. Perea J. "Evaluación dinámico sistémica de la Teoría de Regulación de Recursos Comunes como explicación de su depredación en Colombia”. Bucaramanga, 
Colombia, 2005. Trabajo de grado (Ingeniería de

Sistemas).UNAB. Facultad de Ingeniería de Sistemas.

\section{APÉNDICES}

APENDICE 1. Arquetipo del Mecanismo de Regulación de Privatización [4].

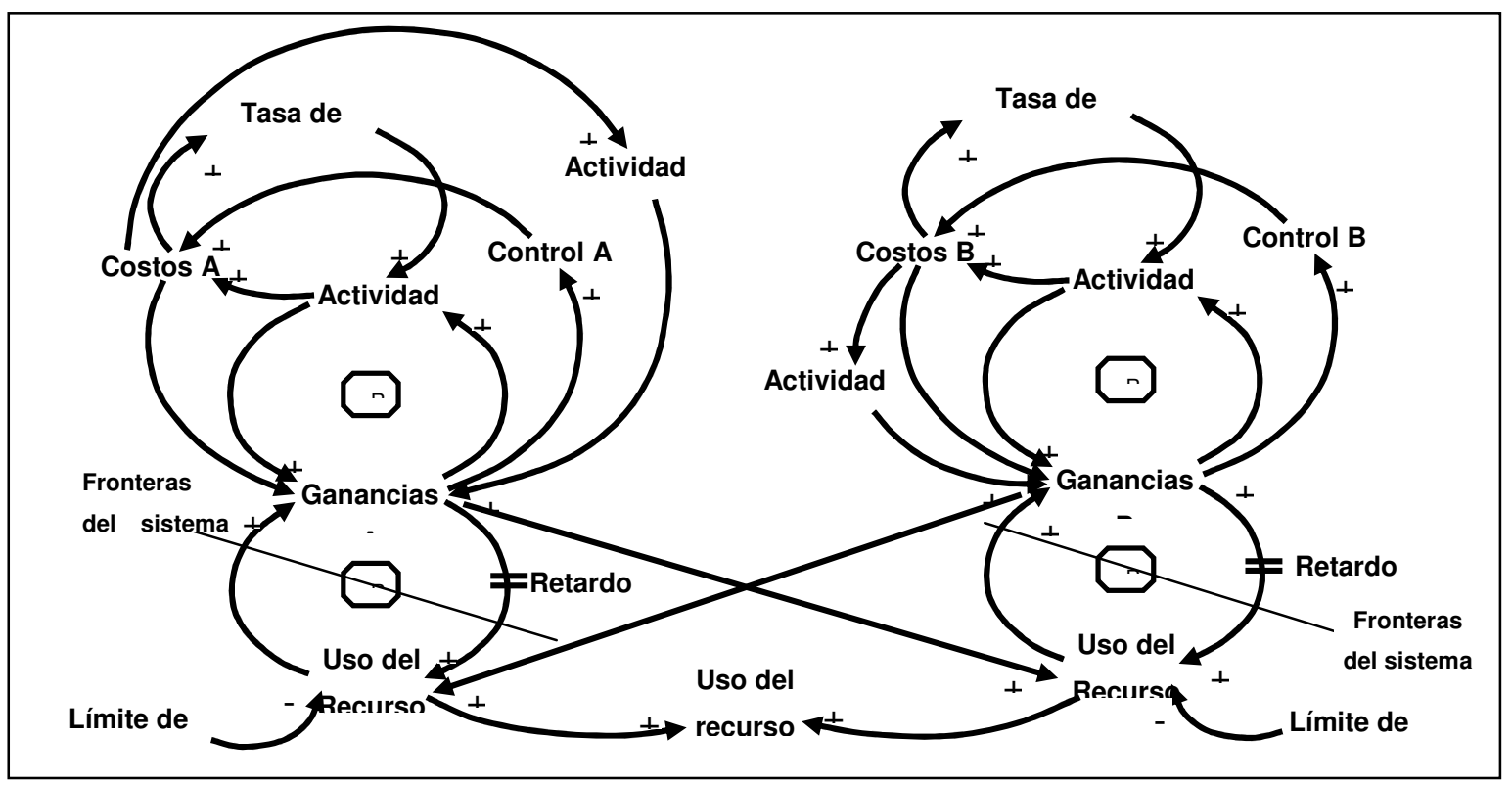

APENDICE 2. Principales ciclos de la dinámica de apropiación del bagre regulado por la privatización [4].

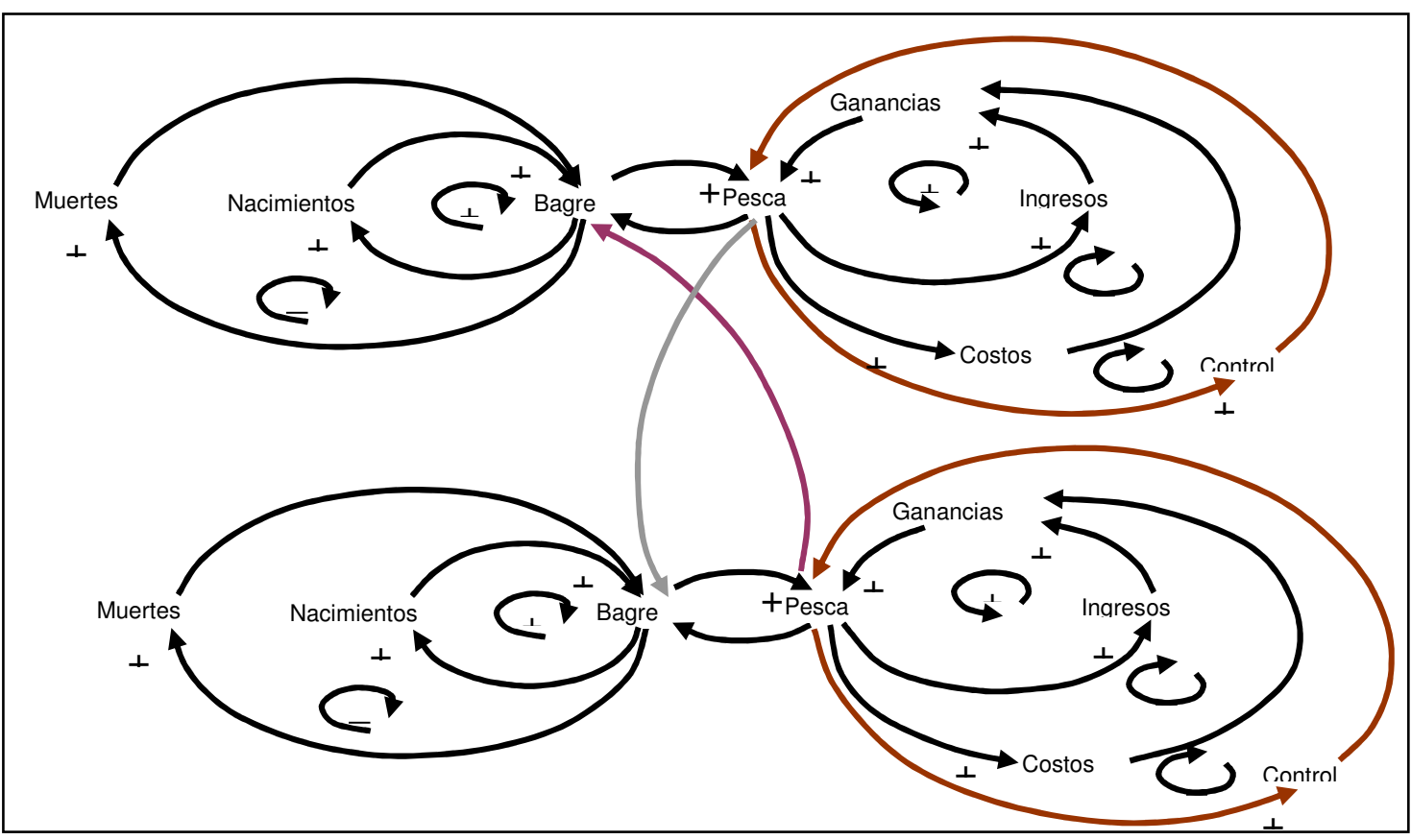

